\section{Expression level of quiescin sulfhydryl oxidase 1 (QSOX1) in neuroblastomas}

\author{
D.G.B. Araújo, ${ }^{1}$ L. Nakao, ${ }^{2}$ P. Gozzo, ${ }^{1}$ \\ C.D.A. Souza, ${ }^{1}$ V. Balderrama, ${ }^{1}$ \\ E.S. Gugelmin, ${ }^{3}$ A.P. Kuczynski, ${ }^{4}$ \\ M. Olandoski, ${ }^{1}$ L. de Noronha ${ }^{1}$
}

'School of Medicine, Pontifical Catholic

University of Paraná, Curitiba-PR

2Department of Basic Pathology, Federal

University of Paraná, Curitiba-PR

${ }^{3}$ Department of Pediatric Pathology,

Pequeno Príncipe Children's Hospital,

Curitiba-PR

${ }^{4}$ Department of Pediatric Oncology,

Pequeno Príncipe Children's Hospital,

Curitiba-PR, Brazil

\section{Abstract}

Neuroblastoma is the most common extracranial solid malignant tumor observed during childhood. Although these tumors can sometimes regress spontaneously or respond well to treatment in infants, genetic alterations that influence apoptosis can, in some cases, confer resistance to chemotherapy or result in relapses and adversely affect prognosis for these patients. The aim of this study was to correlate immunohistochemical expression of the protein quiescin sulfhydryl oxidase 1 (QSOX1) in samples obtained from untreated neuroblastomas with the patients' clinical and pathological prognostic factors and clinical course. Neuroblastoma samples $(\mathrm{n}=23)$ obtained from histology blocks were arrayed into tissue microarrays and analysed by immunohistochemistry. The cases were classified according to the following clinical and pathological prognostic factors: age at diagnosis greater or less than/equal to 18 months; location of the lesion at diagnosis (abdominal or extra-abdominal); presence or absence of bone-marrow infiltration; tumor differentiation (well or poorly differentiated); Shimada histopathologic classification (favourable or unfavourable); state of the tumor extracellular matrix (Schwannian-stroma rich or poor); amplification of the MYCN oncogene; and clinical course (dead or alive with or without relapses/residual lesions). Twelve of the cases were female, 9 children were over 18 months old, 9 cases presented with extra-abdominal tumors and 9 cases exhibited tumors with unfavourable histologies. Fifteen patients underwent bone-marrow biopsy, and 4 of these were positive for metastasis. Nine patients died. The higher immunohistochemical expression of QSOX1 was more common in well-differentiated samples $(\mathrm{P}=0.029)$, in stroma-rich samples $(\mathrm{P}=0.029)$ and in samples from patients with a high prevalence of relapses/residual disease. The functions of QSOX1 include extracellular matrix maturation and the induction of apoptosis. Therefore, QSOX1 may be involved in neuroblastoma differentiation and regression and may thus function as a biomarker for identifying risk groups for this neoplasm.

\section{Introduction}

Neuroblastoma is a neoplasm comprising embryonic nerve cells and is most commonly diagnosed in lactating infants less than 1 year old. It is also the most commonly observed solid extracranial tumor during childhood. ${ }^{1}$

The varied behaviour of neuroblastoma, which is sometimes very aggressive, suggests that the malignant transformation in this disease is at least partially due to a failure to regulate cell differentiation and proliferation. Neuroblastoma tumors sometimes exhibit relatively high benign differentiation rates with spontaneous regression, a phenomenon that may reflect alterations in cell differentiation programs and apoptotic cascades. Neuroblastoma maturation is a well-known process and generally occurs in infants less than 1 year of age with a favourable histology. It is known to be highly dependent on the remodelling of the extracellular matrix (i.e., remodelling of the stroma in neuroblastomas), which evolves from a poorly differentiated neuropil to one that is rich in well-differentiated Schwann cells. ${ }^{1-3}$ Of high interest are those rare patients who, despite their favourable clinical and pathological profiles, exhibit early relapses and a very poor clinical course. Identifying these patients at diagnosis enables more aggressive therapy to be undertaken, even when the good prognostic factors suggest that this course of action is not required, thus improving the chances of patient survival. The quiescin sulfhydryl oxidase (QSOX) proteins are FAD-dependent sulfhydryl oxidases that are found in the extracellular environment (e.g., seminal fluid, chicken egg white and supernatant of quiescent fibroblasts). ${ }^{4-8}$ The extracellular location of QSOX proteins suggests that they may be involved in the remodelling of the extracellular matrix, particularly because QSOX can catalyse the formation of disulphide bridges, which are needed for the appropriate folding and stability of various matrix proteins. QSOX has been observed in the endoplasmic reticulum, Golgi complex, secretory granules, mitochondria and other organelles. $^{9-13}$ Studies using a polyclonal
Corrispondence: Lucia de Noronha, Pontifical Catholic University of Paraná, School of Medicine, Rua Imaculada Conceição, 1155 Prado Velho, 80215-901 Curitiba-PR, Brazil. Tel. +55.41.32712264 - Fax: +55.41 .32711621$ E-mail: Inno@terra.com.br

Keywords: neuroblastoma, QSOX1, proliferation, apoptosis, prognostic factors.

Conflict of interests: the authors declare no conflict of interests.

Contributions: DGBA, ESG, acquisition, analysis and data interpretation; LN, substantial contributions to conception and design; PG, CDAS, VB, acquisition of data; APK, critical revisions; MO, analysis and data interpretation; LN, final approval of the version to be published.

Received for publication: 23 July 2013.

Accepted for publication: 23 January 2014.

This work is licensed under a Creative Commons Attribution NonCommercial 3.0 License (CC BYNC 3.0).

(C) Copyright D.G.B. Araújo et al., 2014

Licensee PAGEPress, Italy

European Journal of Histochemistry 2014; 58:2228 doi:10.4081/ejh.2014.2228

antiQSOX1 antibody in rats revealed the presence of this protein in all areas of the brain. In addition, QSOX2 is reportedly involved in the sensitisation of neuroblastoma cells to apoptotic stimuli and may act as a regulatory factor in cell growth and adhesion. ${ }^{13-17}$

The hypothesis of this study is based on the observation that the expression of QSOX1 in neuroblastoma tumors may influence its clinical course because this protein is involved in processes such as the maturation of the extracellular matrix and the induction of apoptosis in these tumors. These processes are responsible for the differentiation and maturation of these tumors and, consequently, their more benign behaviour. The aim of this study was to investigate the immunoexpression of QSOX1 in human neuroblastoma tumors such that this protein could be used as a prognostic biomarker to help better discriminate among risk groups.

\section{Materials and Methods}

This project was approved by the Committee for Ethics in Research at the Pontifical Catholic University of Paraná (PUCPR) and was conducted according to the principles of the Declaration of Helsinki. All male and female patients at the Pequeno Príncipe 
Children's Hospital with a diagnosis of neuroblastoma were selected for the study. In total, 23 paraffin-embedded neuroblastoma samples were obtained from patients who had not yet been treated.

Each sample was classified as follows: i) gender of the child; ii) age range at diagnosis (less than/equal to 18 months or older than 18 months); iii) location of lesions at diagnosis (extra-abdominal or abdominal); iv) state of bone marrow (presence or absence of neoplastic cell infiltration); v) histological differentiation of the tumor (classified into 2 groups: a) poorly differentiated or undifferentiated tumors; and b) well-differentiated or differentiating neuroblastomas and ganglioneuroblastomas); vi) clinical course of the disease (patient was deceased or alive; if alive, the presence or absence of relapses or residual disease); vii) type of extracellular matrix in the tumor (Schwannian-stroma rich or poor); viii) Shimada classification (favourable or unfavourable histology); ix) staging according to the International Neuroblastoma Staging System (I, IIA, IIB, III, IV or IVS); and x) amplification status of the MYCN oncogene (paraffin embedded-formalin fixed FISH reactions were performed at diagnosis, and these data were obtained).

Two representative areas of the tumor were transferred from the histology block to a recipient tissue microarray (TMA) block. Next, two 4 - $\mu$ m-thick paraffin-embedded sections of the TMA blocks were transferred to electrically charged Star Frost® (Braunschweig, Germany) slides and incubated with a primary anti-QSOX1 recombinant mouse antibody produced at the Federal University of Paraná ${ }^{18,19}$ for $12 \mathrm{~h}$ in a humidified chamber at a temperature between 2 and $8^{\circ} \mathrm{C}$. An Advance Dako® (Carpinteria, CA, USA) secondary antibody was incubated with the slides for $30 \mathrm{~min}$ at a temperature between 2 and $8^{\circ} \mathrm{C}$. The immune reactions were developed by adding DAB chromogen-substrate solution (Dako) to the slides. Harris hematoxylin was used for counterstaining. Positive and negative controls were run in parallel with all reactions.

The slides were read using the Image Pro Plus ${ }^{\circledR}$ (Rockville, MD, USA) software with aid of a Dino-eye ${ }^{\circledR}$ (Taiwan) camera and an Olympus BX40 optical microscope (Japan) (40x objective). The cytoplasm of neuroblastoma cells and extracellular matrix appeared brown when the anti-QSOX1 reaction was positive (Figure 1). A photomicrograph of a high power field $(\mathrm{HPF}=400 \mathrm{x})$ in a positive control slide (human seminal vesicle) was taken. A sample of the positive brown staining pattern from this photomicrograph was subsequently input into the program for the software to automatically identify all of the other positive areas of the photographed field. The photomi- crograph then became the mask used to read all of the slides. Ten HPFs were photographed for each slide, and the previously prepared mask was superimposed on these photomicrographs. Based on the mask, the program automatically measured the total immunopositive area for each HPF in $\mu^{2}$. The average of the 10 immunopositive areas corresponded to the mean immunopositivity (per HPF) for QSOX1 for each neuroblastoma in the study. To compare the groups defined by the clinical and pathological variables and the clinical course of the disease in terms of the quantitative variables, Student's $t$-test or a nonparametric Mann-Whitney test was used. Fisher's exact test was used to compare the groups in terms of dichotomous nominal variables. Patient survival was analysed using Kaplan-Meier curves, and the log-rank test was used to compare pairs of curves. A significance level of $\mathrm{P}<0.05$ was used. The statistical analysis program used was SPSSV8 ${ }^{\circledR}$. To determine the cut-off points that best represented the correlation between the 2 groups, the ROC curves were adjusted. The cut-off points were determined using the groups with or without relapse/residual disease; the value of the area immunopositive for QSOX1 (in $\mu \mathrm{m}^{2}$ ) that best distinguished between the 2 groups was calculated, and the best product (sensitivity $\mathrm{x}$ specificity)
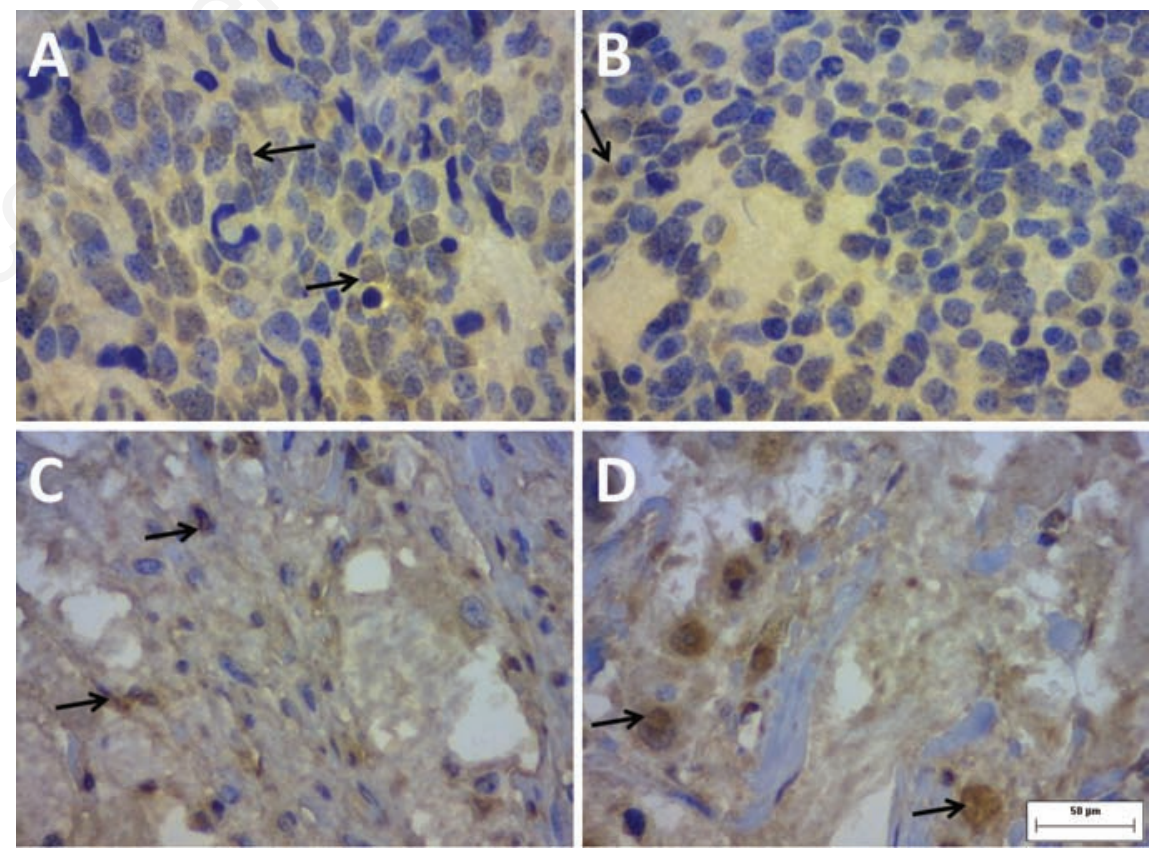

Figure 1. Immunohistochemical staining of the biomarker QSOX1 in neuroblastoma slides $(400 \mathrm{x})$. Note the positive staining in the cytoplasm, the extracellular matrix and, sometimes, the perinuclear space. The staining in the extracellular matrix is weaker in $\mathrm{A}$ ) and B) and is more intense in C) and D). A) and B), poorly differentiated, Schwannianstroma poor neuroblastoma with unfavourable Shimada classification and low immunopositivity for QSOX1 $\left(\leq 65 \mu \mathrm{m}^{2}\right)$. C) and D), well-differentiated, Schwannianstroma rich neuroblastoma with favourable Shimada classification and high immunopositivity for QSOX1 $\left(\geq 65 \mu^{2}\right)$. was considered. After the ROC curve was calculated, the patients were divided into 2 groups according to the immunopositivity immunopositivity (greater than $65 \mu^{2}$ ) and (b) group with low immunopositivity (less than or equal to $65 \mu \mathrm{m}^{2}$ ).

Statistical analyses of prognosis (relapses and/or residual disease) and/or survival (dead or alive) using sensitivity, specificity, positive and negative predictive values for QSOX1 tion were performed.

\section{Results}

There was no significant predominance of either sex. The majority of the patients were diagnosed with neuroblastoma before the age of 18 months (60.8\%). Most of the lesions were located in the abdomen (60.8\%) and exhibited
well-differentiated histology (60.8\%). Only $65 \%$ underwent a bone marrow biopsy, and in been infiltrated by the tumor. At diagnosis, 5
patients were stage IIA, 2 were stage III and 16 were stage IV. In the Shimada classification, 18 Schwannian-stroma and Shimada classifica$26.6 \%$ of these cases, the bone marrow had 
patients had favourable histologies, and 5 exhibited unfavourable histologies; 14 patients had Schwannian stroma-rich tumors, and 9 exhibited Schwannian stroma-poor tumors. Three years after diagnosis, $60 \%$ of the patients were still alive, although in $57 \%$ of these cases the disease remained active (Table 1). None of the cases studied exhibited an amplification of MYCN.

The immunoexpression of QSOX1 correlated with the type of tumor stroma (Figure 1) and was higher in Schwannian stroma-rich tumors, with a tendency towards statistical significance $(\mathrm{P}=0.054)$ (Table 1$)$. Using the cutoff point established from the ROC curve, new comparisons were made among the groups defined by the clinical, pathological and clinical-course variables (Table 2). The data in

Table 1. Correlation between the results for mean QSOX1 expression and the clinical and pathological variables.

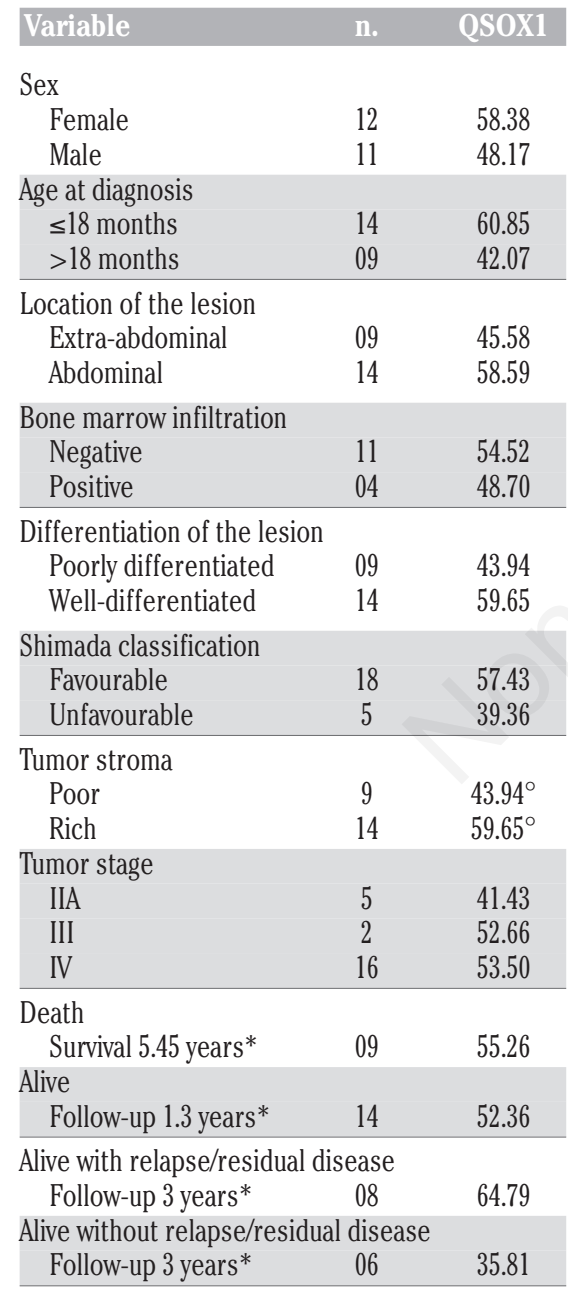

Mean QSOX1 values are expressed as the mean positive staining area in $\mu \mathrm{m}^{2}$ per histological field. *Follow-up time or mean survival in years for each of the clinical-course variables, i.e. whether the patient died or was alive with or without a relapse/residual disease. ${ }^{\circ} \mathrm{P}=0.054$, with a statistically significant trend.
Table 2 exhibit a statistically significant correlation between QSOX1 expression and well-differentiated neuroblastomas $(\mathrm{P}=0.029)$. Table 2 also shows that there was a correlation between high immunohistochemical expression of QSOX1 and stroma-rich samples $(\mathrm{P}=0.029)$.

There was no statistically significant correlation between the number of deaths and the clinical and pathological variables or between the number of deaths and the expression of QSOX1. Nine patients exhibited low immunopositivity $\left(\leq 65 \mu^{2}\right), 3$ of whom exhibited relapses/residual disease and 6 of whom did not show signs of relapse/residual disease (Table 3). Of the 5 who showed higher immunopositivity for QSOX1 $\left(>65 \mu \mathrm{m}^{2}\right)$, all of them exhibited relapses/residual disease $\mathrm{P}=0.031$ ). Therefore, more instances of relapse/residual disease occurred among cases with immunopositive areas greater than 65 $\mu^{2}$ (i.e., higher expression of QSOX1). There was no statistically significant correlation between the death rate and expression of QSOX1.

A Cox regression model was adjusted to analyse the relationship between survival time and clinical and pathological variables and the area that was immunopositive for QSOX1. The results indicated that there was no significant association between the variables included in the model and the patient survival curve. The correlation between prognosis (relapses and/or residual disease) and/or survival (dead or alive) with QSOX1 expression demonstrated that a higher expression of QSOX1 may be associated with increasingly poor prognoses and survival (higher specificity and positive predictive value) (Table 4).

\section{Discussion}

QSOX1 is an enzyme that is thought to promote the maturation of the extracellular matrix because it is involved in many extracellular processes, including the formation of disulphide bridges. It also participates in redox reactions and contributes to increased cellular oxidative stress and apoptosis induction, which has been observed in fibroblasts and progenitor cells isolated from the peripheral nervous system. QSOX1 is also thought to play a role in neurodegenerative processes because it is associated with dysfunctions in neuronal cell growth and differentiation, maturation of glial cells and production of growth factors. ${ }^{18-23 .}$ In human neuroblastoma, QSOX2 appears to be involved in the apoptosis of tumor cells. ${ }^{17}$

Table 2. Correlation between the marker QSOX1 and the clinical and pathological variables studied using the cut-off point selected by ROC curve analysis; $>65 \mu^{2}$ per HPF indicates the presence of relapses.

$\begin{array}{ccc}\text { Variable } & \text { Immunopositivity for QSOX1 Total } & \text { P } \\ & \leq 65 \text { (low) } & >65 \text { (high) }\end{array}$

Age at diagnosis

\begin{tabular}{lllll}
$\leq 18$ months & $08(57.14 \%)$ & $06(42.86 \%)$ & 14 & \\
$>18$ months & $05(55.56 \%)$ & $04(44.44 \%)$ & 09 & 1.000 \\
Location of the lesion & & & & \\
Extra-abdominal & $07(77.78 \%)$ & $02(22.22 \%)$ & 09 & \\
Abdominal & $06(42.86 \%)$ & $08(57.14 \%)$ & 14 & 0.197 \\
\hline
\end{tabular}

Bone marrow infiltration

$\begin{array}{lllll}\text { Negative } & 06(54.55 \%) & 05(45.45 \%) & 11 & \\ \text { Positive } & 03(75.00 \%) & 01(25.00 \%) & 04 & 0.604\end{array}$

\begin{tabular}{lllll} 
Shimada classification & & & \\
Unfavourable & $04(80.00 \%)$ & $01(20.00 \%)$ & 05 & \\
Favourable & $09(50.00 \%)$ & $09(50.00 \%)$ & 18 & 0.339 \\
\hline
\end{tabular}

\begin{tabular}{lcccc}
\hline $\begin{array}{l}\text { Stroma } \\
\text { Rich }\end{array}$ & & & & \\
Poor & $08(35.71 \%)$ & $09(64.29 \%)$ & 14 & \\
Tumor stage & & $01(11.11 \%)$ & 9 & $0.029^{\circ}$ \\
IIA & $3(60.00 \%)$ & $2(40.00 \%)$ & 5 & \\
III & $2(100.00 \%)$ & $0(00.00 \%)$ & 2 & \\
IV or IVS & $8(50.00 \%)$ & $8(50.00 \%)$ & 16 & 0.405 \\
\hline $\begin{array}{l}\text { Differentiation of the lesion } \\
\text { Poorly differentiated }\end{array}$ & $08(88.89 \%)$ & $01(11.11 \%)$ & 09 & \\
Well-differentiated & $05(35.71 \%)$ & $09(64.29 \%)$ & 14 & $0.029^{\circ}$ \\
\hline
\end{tabular}

*Fisher's exact test, $\mathrm{P}<0.05$. Mean immunopositivity for QSOX1 for each sample was measured in $\mu \mathrm{m}^{2}$ per high-power field. ${ }^{\circ}$ Statistically significant values. 
We have found scarce evidence to support this claim in the literature, and we believe that QSOX1 may play a role in both the apoptosis/proliferation of and extracellular matrix-cell adhesion/maturation in neuroblastomas. ${ }^{22,24-26}$

Since QSOX1 is involved in cell regeneration, maturation of the extracellular matrix (especially the neural extracellular matrix) and apoptosis, tumor samples that have histological patterns and/or clinical and pathological factors associated with a better prognosis should exhibit higher QSOX1 immunopositivity due to the close relationship between neuroblastoma differentiation, changes in extracellular matrix maturation and increases in apoptotic rates. In our study population, there was a direct and statistically significant corre- lation between the higher immunoexpression of QSOX1 and the presence of well-differentiated stroma-rich tumors. This protein could therefore act as a biomarker of differentiation and favourable evolution of the lesion. However, contrary to our expectations, most of the samples obtained from the group with relapses/residual neoplasms exhibited higher expression of this protein, whereas all of the samples from the group without relapses/ residual neoplasms exhibited low expression (Table 3). Moreover, higher QSOX1 expression $\left(>65 \mu^{2}\right.$ ) correlated with poor prognosis and/or low survival rates, and its expression was associated with high rates of specificity and positive predictive values (Table 4). This finding can be explained by the observation that the vast majority of these patients did not have relapses but instead exhibited residual disease following chemotherapy. Chemotherapy for neuroblastoma is generally more effective in less differentiated areas of the tumor and spares the more differentiated areas, which may subsequently appear as residual disease. Therefore, it is likely that a greater prevalence of residual disease would be observed in patients with tumors that have large well-differentiated areas; consequently, these patients would exhibit higher QSOX1 expression. Moreover, it should be noted that the number of cases studied in this report is small; thus, the confidence intervals are quite large (Table 4). However, we should consider that higher QSOX1 expression may be related to poor prognosis, though we observed its correlation with good prognostic factors such as

Table 3. Correlation between the marker QSOX1 and the presence or absence of relapse/residual disease or death using the cut-off point selected by ROC curve analysis; $>65 \mu \mathrm{m}^{2}$ per HPF indicates the presence of relapse/residual disease.

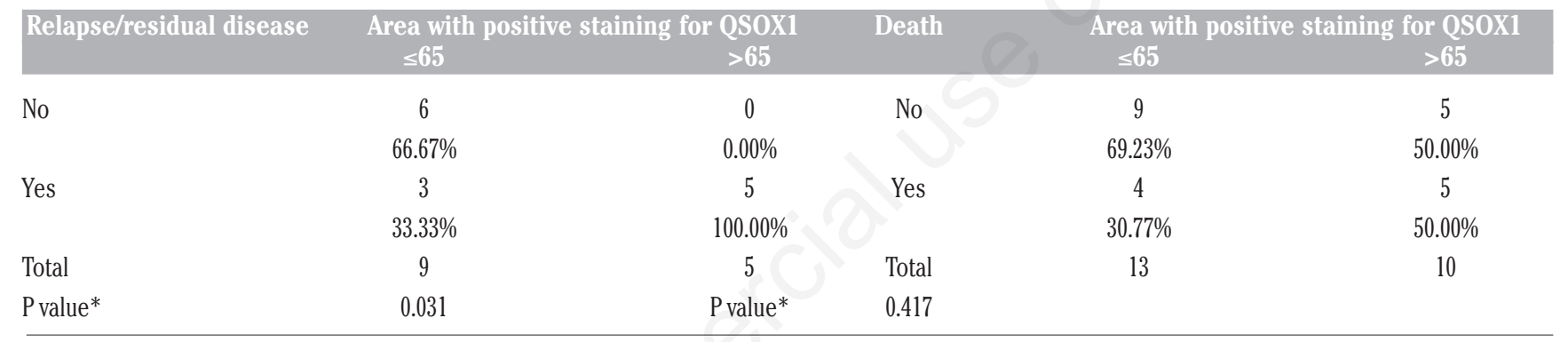

*Fisher's exact test, $\mathrm{P}<0.05$. The mean immunopositivity for QSOX1 for each study sample was measured in $\mu \mathrm{m}^{2}$ per high-power field.

Table 4. Correlation between relapse/residual disease (prognosis) and/or death (survival) with QSOX1 expression, tumor differentiation, tumor stroma pattern and Shimada classification. Sensitivity, specificity, positive predictive value and negative predictive value were determined.

\begin{tabular}{|c|c|c|c|c|c|c|}
\hline & $\begin{array}{r}\text { Re } \\
\text { residua } \\
\text { or }\end{array}$ & e and & Total & P value & Quality score & $\begin{array}{c}\text { Results } \\
\text { (confidence intervals) }\end{array}$ \\
\hline & Yes & No & & & & \\
\hline QSOX $>65$ & $10(58.8 \%)$ & $0(0 \%)$ & 10 & & Sens & $58.8(35.4-82.2)$ \\
\hline QSOX $\leq 65$ & $7(41.2 \%)$ & $6(100 \%)$ & 13 & 0.019 & Spec & $100(100-100)$ \\
\hline & & & & & PPV & $100(100-100$ \\
\hline Total & 17 & 6 & 23 & & NPV & $46.2(19.1-73.3)$ \\
\hline Poor-differentiated tumor & $5(29.4 \%)$ & $4(66.7 \%)$ & 9 & & Sens & $29.4(7.8-51.1)$ \\
\hline Well-differentiated tumor & $12(70.6 \%)$ & $2(33.3 \%)$ & 14 & 0.162 & $\begin{array}{l}\text { Spec } \\
\text { PPV }\end{array}$ & $33.3(0-71.1)$ \\
\hline Total & 17 & 6 & 23 & & NPV & $\begin{array}{l}14.3(0-32.6) \\
\end{array}$ \\
\hline Stroma-poor tumor & $5(29.4 \%)$ & $4(66.7 \%)$ & 9 & & Sens & $29.4(7.8-51.1)$ \\
\hline Stroma-rich tumor & $12(70.6 \%)$ & $2(33.3 \%)$ & 14 & 0.162 & $\begin{array}{l}\text { Spec } \\
\text { PPV }\end{array}$ & $\begin{array}{c}33.3(0-71.1) \\
55.6(23.1-88.0)\end{array}$ \\
\hline Total & 17 & 6 & 23 & & NPV & $14.3(0-32.6)$ \\
\hline Shimada unfavourable & $4(23.5 \%)$ & $1(16.7 \%)$ & 5 & & Sens & $23.5(3.4-43.7)$ \\
\hline Shimada favourable & $13(76.5 \%)$ & $5(83.3 \%)$ & 18 & 1 & $\begin{array}{l}\text { Spec } \\
\text { PPV }\end{array}$ & $\begin{array}{c}83.3(53.5-100) \\
80(44.9-100)\end{array}$ \\
\hline Total & 17 & 6 & 23 & & NPV & $27.8(7.1-48.5)$ \\
\hline
\end{tabular}

Sens, sensitivity: $\mathrm{P}\left(\mathrm{QSOX}>65 \mu \mathrm{m}^{2}\right.$ as occurs in relapse/residual disease and/or death); Spec, specificity: $\mathrm{P}\left(\mathrm{QSOX} \leq 65 \mu \mathrm{m}^{2}\right.$ because there is relapse/residual disease and/or death); PPV, positive predictive value: $\mathrm{P}$ (a relapse/residual disease and/or death as $\mathrm{QSOX}>65 \mu \mathrm{m}^{2}$ ); NPV, negative predictive value: $\mathrm{P}$ (not a relapse/residual disease and/or death as $\mathrm{QSOX} \leq 65 \mu \mathrm{m}^{2}$ ). Mean immunopositivity for QSOX1 for each sample was measured in $\mu \mathrm{m}^{2}$ per high-power field. ${ }^{\circ}$ Statistically significant value. 
well-differentiated tumors and stroma-rich tumors in this study. The reason for this consideration is that these 2 factors are related to tumors with large amounts of stroma and therefore exhibit much higher QSOX1 expression, independent of biological behaviour. However, these last 2 characteristics should be considered together because the samples that qualified as well-differentiated are the same samples that are defined as stroma-rich (the $p$ values for these 2 groups are the same). Other authors have previously described the association of higher QSOX1 expression with a higher risk of recurrence and poor prognosis in breast cancer. ${ }^{22,24,25}$

Regardless of QSOX1's involvement in maturation of the neural extracellular matrix and apoptosis and given that neuroblastoma maturation/differentiation is well correlated with survival, this work suggests a lack of correlation between the QSOX1 data and patient survival. This observation could be related to a delay in diagnosis because this is very common in Brazil: alternatively, it could be related to very important differences among treatment protocols due to the presentation of these patients. Another reason could be the very small sample number of cases used to obtain good survival curves, which is a limitation of this study. The higher immunohistochemical expression of QSOX1 was more common in well-differentiated samples $(\mathrm{P}=0.029)$, in stroma-rich samples $(\mathrm{P}=0.029)$ and in samples from patients with a high prevalence of relapses/residual disease.

\section{References}

1. Kushner BH, Cheung NV. Neuroblastoma from genetic profiles to clinical challenge. N Engl J Med 2005;353:2215-17.

2. Islam A, Kageyama H, Takada N, Kawamoto T, Takayasu H, Isogai E, et al. High expression of survivin, mapped to $17 q 25$, is significantly associated with poor prognostic factors and promotes cell survival in human neuroblastoma. Oncogene 2000;19:617-23.

3. Casciano II, Mazzocco K, Boni L, Pagnan G, Banelli B, Allemanni G, et al. Expression of DeltaNp73 is a molecular marker for adverse outcome in neuroblastoma patients. Cell Death Differ 2002;9:246-51.

4. Thorpe C, Hoober KL, Raje S, Glynn NM, Burnside J, Turi GK, et al. Sulfhydryl oxi- dases: emerging catalysts of protein disulfide bond formation in eukaryotes. Arch Biochem Biophys 2002;405:1-12.

5. Morel C, Adami P, Musard J-F, Duval D, Radom J, Jouvenot M. Involvement of sulfhydryl oxidase QSOX1 in the protection of cells against oxidative stressinduced apoptosis. Exp Cell Res 2007;313: 3971-82.

6. Chakravarthi S, Jessop CE, Willer M, Stirling CJ, Bulleid NJ. Intracellular catalysis of disulfide bond formation by the human sulfhydryl oxidase, QSOX1. Biochem J 2007;404:403-11.

7. Inaba K. Structural basis of protein disulfide bond generation in the cell. Genes Cells 2010;15:935-43.

8. Heckler EJ, Rancy PC, Kodali VK, Thorpe C. Generating disulfides with the quiescin sulfhydryl oxidases. Biochim Biophys Acta 2008;1783:567-77.

9. Margittai E, Sitia R. Oxidative protein folding in the secretory pathway and redox signaling across compartments and cells. Traffic 2011;12:1-8.

10. Malinouski M, Zhou Y, Belousov VV, Hatfield DL, Gladyshev VN. Hydrogen peroxide probes directed to different cellular compartments. Plos One 2011;6:1-10.

11. Heckler EJ, Alon A, Fass D, Thorpe C. Human quiescin-sulfhydryl oxidase, QSOX1: probing internal redox steps by mutagenesis. Biochemistry 2008;47:4955-63.

12. Benayoun B, Esnard-Fève A, Castella S, Courty Y, Esnard F. Rat seminal vesicle FAD-dependent sulfhydryl oxidase. J Biol Chem 2001;276:13830-37.

13. Hoober KL, Joneja B, White HB 3rd, Thorpe C. A Sulfhydryl oxidase from chichen egg white. Biol Chem 1996;271: 30510-16.

14. Hoober KL, Glynn NM, Burnside J, Coppock DL, Thorpe C. Homology between egg white sulfhydryl oxidase and quiescin Q6 defines a new class of flavin-linked sulfhydryl oxidases. J Biol Chem 1999;274: 31759-62.

15. Coppock DL, Kopman C, Scandalis S, Gilleran S. Preferential gene expression in quiescent human lung fibroblasts. Cell Growth Differ 1993;4:483-93.

16. Coppock DL, Cina-Poppe D, Gilleran S, et al. The quiescin Q6 gene (QSCN6) is a fusion of two ancient gene families: thioredoxin and ERV1. Genomics 1998;54:460-8.

17. Wittke I, Wiedemeyer R, Pillmann A,
Savelyeva L, Westermann F, Schwab M. Neuroblastoma-derived sulfhydryl oxidase, a new member of the sulfhydryl oxidase/quiescin6 family, regulates sensitization to interferon gamma-induced cell death in human neuroblastoma cells. Cancer Res 2003;15:7742-52.

18. Portes KF, Ikegami CM, Getz J, Martins AP, Noronha L, Zischler LF, et al. Tissue distribution of quiescin Q6/sulfhydryl oxidase (QSOX) in developing mouse. J Mol 2008; 39:217-25.

19. Zanata SM, Luvizon AC, Batista DF, Ikegami CM, Pedrosa FO, Souza EM, et al. High levels of active quiescin Q6 sulfhydryl oxidase (QSOX) are selectively present in fetal serum. Redox Report (Edinburgh) 2005;10:6.

20. Morel C, Adami P, Musard JF, Duval D, Radom J, Jouvenot M. Involvement of sulfhydryl oxidase QSOX1 in the protection of cells against oxidative stressinduced apoptosis. Exp Cell Res 2007;313: 3971-82.

21. Coppock D, Kopman C, Scandalis S, Guileran S. Preferential gene expression in quiescent human lung fibroblasts. Cell Growth Differ 1991;4:483-91.

22. Ilany T, Alon A, Grossman I, Horowitz B, Kartvelishvily E, et al. A secreted disulfide catalyst extracellular matrix composition and function. Science 2013;341:74-6.

23. Amiot C, Musard JF, Hadjiyiassemis M, Jouvenot M, Fellmann D, Risold PY, et al. Expression of the secreted FAD-dependent sulfydryloxidase (QSOX) in the guinea pig central nervous system. Mol Brain Res 2004;125:13-21.

24. Soloviev M, Esteves, MP, Amiri F, Crompton MR, Rider CC. Elevated transcription of the gene QSOX1 encoding quiescin Q6 sulfhydryl oxidase 1 in breast cancer. PLoS One 2013;8:e57327.

25. Katchman BA, Ocal T, Cinliffe HE, Chang $\mathrm{Y}$, Hostetter $\mathrm{G}$ et al. Ezpression of quiescin sulfhydryl oxidase 1 is associated with a highly invasive phenotype and correlates with a poor prognosis in luminal $B$ breast cancer. Breast Cancer Res 2013;15:R28.

26. Noronha L, Araújo DGB, Gozzo PC, Harada LE, Percicote AP et al. Immunoexpression of cell cycle biomarkers in neuroblastoma samples and its correlation with prognostic factors. J. Bras. Patol Med Lab 2013;49: $57-63$. 